\title{
A Giant Bipolar Shell around the WO star in the Galaxy IC 1613: Structure and Kinematics ${ }^{1}$
}

\author{
Afanasiev V.L. ${ }^{a}$, Lozinskaya T.A. ${ }^{b 2}$, Moiseev A.V. ${ }^{a}$, Blanton E. ${ }^{c}$ \\ ${ }^{a}$ Special Astrophysical Observatory of the Russian AS, Nizhnij Arkhyz 357147, Russia \\ ${ }^{b}$ Sternberg Astronomical Institute, Universitetskii pr. 13, Moscow, 119899,Russia \\ ${ }^{c}$ Columbia University, New York, USA
}

\begin{abstract}
Observations of the nebula associated with the WO star in the galaxy IC 1613 are presented. The observations were carried out with a scanning Interferometer Fabry-Perot in $\mathrm{H}_{\alpha}$ at the $6 \mathrm{~m}$ Special Astrophysical Observatory telescope; narrow-band $\mathrm{H}_{\alpha}$ and [OIII] images were obtained with the 4-m KPNO telescope. The monochromatic $\mathrm{H}_{\alpha}$ image clearly reveals a giant bipolar shell structure outside the bright nebula S3. The sizes of the southeastern and northwestern shells are $112 \times 77 \mathrm{pc}$ and $(186-192) \times(214-224) \mathrm{pc}$, respectively. We have studied the object's kinematics for the first time and found evidence for expansion of both shells. The expansion velocities of the southeastern and northwestern shells exceed 50 and $70 \mathrm{~km} \mathrm{~s}^{-1}$, respectively. We revealed a filamentary structure of the shells and several compact features in the S3 core. A scenario is proposed for the formation of the giant bipolar structure by the stellar wind from the central WO star located at the boundary of a dense "supercavity" in the galaxy's H I distribution.
\end{abstract}

\section{Introduction}

Among more than five hundred WR stars in the Local-Group galaxies, only six objects belong to a rare group of the "oxygen" stars Wolf-Rayet (WO). According to Barlow \& Hummer (1982), they can be considered as a separate sequence in the WN-WCWO chain, which represents a very short final evolutionary stage of massive stars close to a bare $\mathrm{CO}$ core. The WO stars are characterizes by a "superwind" (with a velocity up to $5000-6000 \mathrm{~km} \mathrm{~s}^{-1}$ (see Barlow \& Hummer, 1982; Torres et al.,1986; Dopita et al.,1990;Polcaro et al., 1992) preceded by the wind at the MS and WR stages. The WO effective temperature reaches $10^{5} \mathrm{~K}$ (Maeder \& Meynet, 1989;Dopita et al.,1990; Melnik \&Malayeri ;Polcaro et al.,1991).

Of the six WO stars in the Local-Group galaxies, three were identified in our Galaxy, one in the LMC, one in the SMC, and one in the irregular dwarf galaxy IC 1613; only two of them are associated with bright

\footnotetext{
1 Astronomy Letters, Vol.26,No 3, 2000, pp.153-161. Translated from Pis'ma v Astronomicheskii Zhurnal, Vol.26, No. 3, pp. 190-199. Translated by V. Astakhov.

${ }^{2}$ E-mail address for contacts: lozinsk@sai.msu.su
}

nebulae, including WO in IC 1613.

Our interest in the latter object also stems from the fact that WO is the only WR star identified in IC 1613, although the number of WO stars cannot exceed $\approx 1 \%$ of the WR population because of their short lifetime.

D'Odorico \& Rosa (1982) and Davidson \& Kinman (1982) identified the WO star in IC 1613 by broad lines typical of this class of stars in the spectrum of the core of the bright emission nebula S3 (Sandage, 1971). The star's coordinates RA(1950) = $1^{h} 02^{m} 27^{s} 3, \operatorname{DEC}(1950)=+01^{\circ} 48^{\prime} 17^{\prime \prime}$, are known with a $1^{\prime \prime}$ accuracy (Armandroff \& Massey, 1985).

The central part of S3 is characterized by bright HeII 4686 emission (Smith, 1975; D'Odorico \& Rosa, 1982; Garnett et.al, 1991). A detailed spectral analysis and a discussion of the chemical composition of the bright nebula are presented in (Garnet et al., 1991, Kinsburgh \& Barlow, 1995). Narrow-band $\mathrm{H}_{\alpha}$ images revealed regions of weaker emission predominantly southeast of the previously known bright, elongated nebula S3 (Hodge et al., 1990; Hunter et al., 1993).

Thermal radio emission was detected from S3; the sizes of the radio source at half maximum intensity are $19 \times 14^{\prime \prime}$, and the radio image faithfully repro- 
duces the shape of the bright optical nebula (Goss \& Lozinskaya, 1995).

In this paper, we present our $\mathrm{H}_{\alpha}$ observations of the nebula with a Fabry-Perot interferometer on the 6m Special Astrophysical Observatory (SAO) telescope, as well as narrow-band $\mathrm{H}_{\alpha}$ and [OIII] images of the region obtained with the 4-m KPNO telescope.

For the first time, we have clearly revealed a weak outer bipolar shell structure far outside the bright nebula S3 and studied the kinematics of the object.

In Sect. 2, we provide basic parameters of the instruments and describe the observing and reduction techniques; results of the interferometric observations with the $6 \mathrm{~m}$ telescope and the observations with the 4-m KPNO telescope are presented in Sect. 3. The origin of the unique bipolar shell structure of the nebula around the WO star is discussed in Sect. 4.

\section{Observations and data reduction}

\subsection{Interferometric Observations}

The interferometric $\mathrm{H}_{\alpha}$ observations were carried out with a scanning Fabry-Perot interferometer with focal reducer at the prime focus of the $6 \mathrm{~m}$ SAO telescope SAO (the equivalent focus is $\mathrm{F} / 2.4$ ). The interferometer is described in Dodonov et al. (1995). The pre-monochromatization was performed by using an interference filter with the $F W H M=15 \AA$ centered on $\mathrm{H}_{\alpha}$ and tilted at an angle of 6.5 .

We used a Fabry-Perot etalon operating in the 235th order at the $\mathrm{H}_{\alpha}$ wavelength; the separation between adjacent orders $\Delta \lambda=27.9 \AA$ corresponded to the $1274 \mathrm{~km} \mathrm{~s}^{-1}$ range free from order overlapping. The detector was a TK1024 $1024 \times 1024$-pixel CCD array. The observations were carried out with a $2 \times 2$-pixel instrumental binning to reduce the readout time. We obtained $522 \times 522$-pixel images in each spectral channel, with the pixel size corresponding to 0.'68.

The size of the spectral channel was $\delta \lambda=1.16 \AA$ or $53 \mathrm{~km} \mathrm{~s}^{-1}$ in $\mathrm{H}_{\alpha}$. The actual instrumental profile of the interferometer was determined from night-sky lines $6568.8,6577.2 / 6577.4,6562.8 \AA$, and $\mathrm{H}_{\alpha}$ ); the profile width was about $3 \AA$ or $140 \mathrm{~km} \mathrm{~s}^{-1}$.

We performed the observations on August 31, 1998 , with a $2.5-3^{\prime \prime}$ seeing at the zenith distance $z=51-54^{\circ}$. A total of 24 interferograms were obtained for various interferometer plate spacings. The time of a single exposure was $240 \mathrm{~s}$.

The He I (6678.15 $)$ line of a calibration lamp was used for the phase shift measurement. After the observations, we made several accumulations of the images of the caps of the $6 \mathrm{~m}$ primary mirror uniformly illuminated by a lamp of continuous light. This allowed us to make a correction for nonuniform detector sensitivity (flat-fielding) and simultaneously to adjust the filter passband.

The observational data were represented as $522 \times$ $522 \times 24$-pixel "data cubes"; i.e., a 24-channel spectrum corresponded to each image element.

The data reduction (correction for the phase shift, subtraction of the night-sky spectrum, construction of the velocity field and the emission line and continuum images) was carried out by using the ADHOC software package developed by Boulesteix (1993).

Using star images from the Digital Palomar Sky Survey Atlas (DSS), we rotated the original images to coincide with the correct orientation $\alpha-\delta$ and then averaged a $2 \times 2$ element, so that the resulting pixel size was $1^{\prime \prime} 34$.

To increase the signal-to-noise ratio, we performed an optimal filtration of the cube of observational data: smoothing by Gaussian with 1.5 channels FWHM in spectral direction and smoothing in spatial direction with FWHM about of the seeing value. After the smoothing, the resulting spatial resolution was $4^{\prime \prime}$.

Although the actual spectral resolution for the etalon in the 235th order of interference was $130-$ $150 \mathrm{~km} \mathrm{~s}^{-1}$ in $\mathrm{H}_{\alpha}$, the accuracy of measuring the velocity from the line centroid (or from the Gaussian fit) in the case of a symmetrical profile is considerably higher and is determined by the accuracy of phase calibration. The actual accuracy of a single measurement was checked by using a comparison spectrum and was $8-10 \mathrm{~km} \mathrm{~s}^{-1}$.

The tie-in to the absolute velocity was checked by using night-sky lines. The possible systematic shift did not exceed $15-20 \mathrm{~km} \mathrm{~s}^{-1}$.

\subsection{Narrow-Band Images}

The narrow-band $\mathrm{H}_{\alpha}$ and [OIII] images of the nebula were obtained with the 4-m Kitt Peak National Observatory (KPNO) telescope. Interference filters centered on the $\mathrm{H}_{\alpha}(\lambda=6562 \AA, F W H M=29 \AA)$ and [OIII] $(\lambda=5009 \AA, F W H M=45 \AA)$ lines were used. The detector was a $2048 \times 2048$-pixel CCD array with the pixel size corresponding to 0 "' 47 .

The observations were carried out on December 29 and 30, 1995; the angular resolution determined from star images was 1".2 in $\mathrm{H}_{\alpha}$ and 1".4 in [OIII].

\section{Results of the observations}




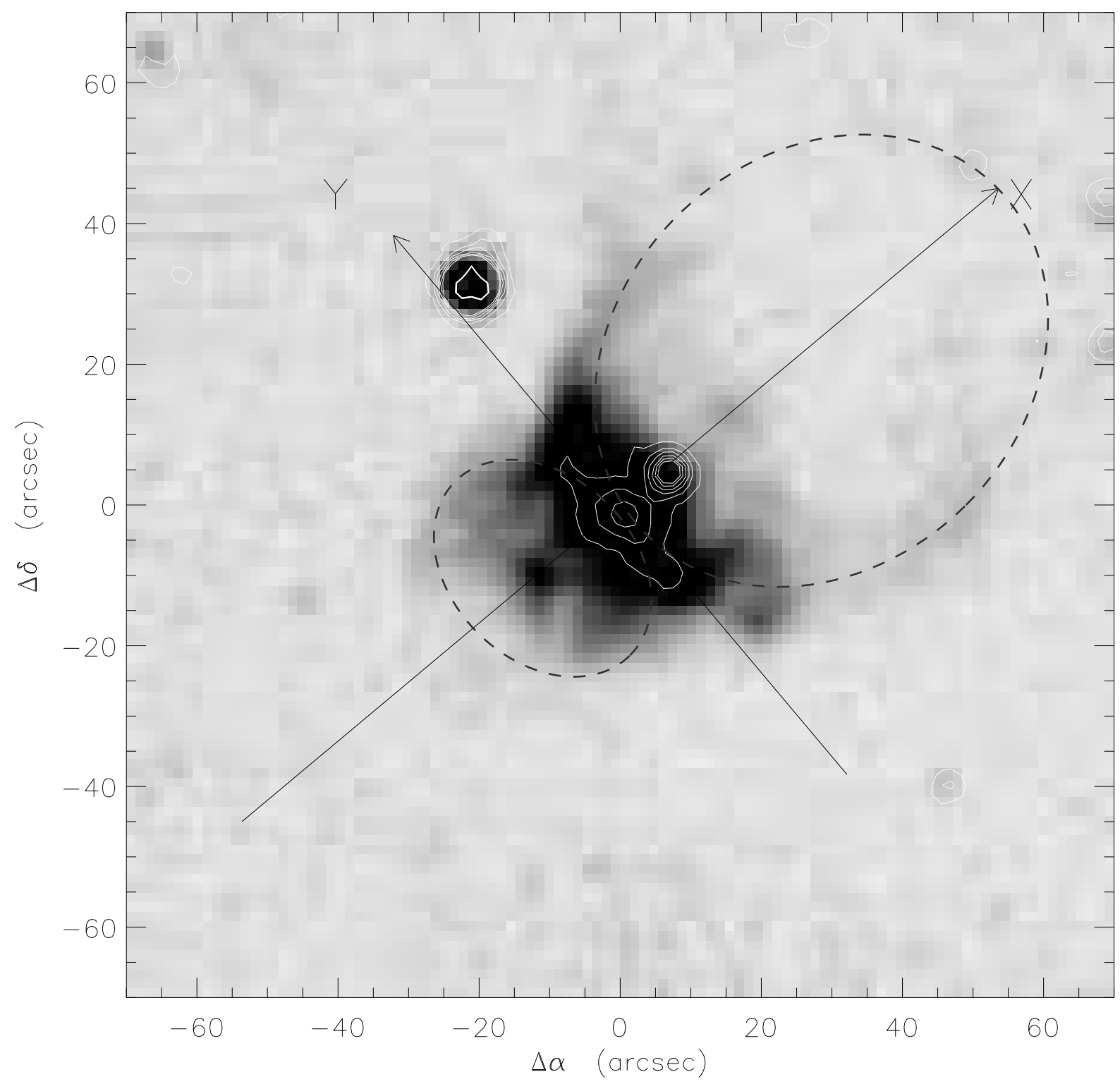

Figure 1: The monochromatic $\mathrm{H}_{\alpha}$ image of the region obtained with Interferometer Fabry-Perot by integrating the emission over all spectral channels of the line. The image of the same region in continuum near $\mathrm{H}_{\alpha}$ line is indicated by isophotes. The dashed line represents the giant bipolar shell.

\subsection{Monochromatic $\mathbf{H}_{\alpha}$ Image}

Figure 1 shows the monochromatic $\mathrm{H}_{\alpha}$ image of the region obtained by integrating the emission over all spectral channels of the line. For comparison, the image of the same region in continuum in the immediate vicinity of $\mathrm{H}_{\alpha}$ line is indicated by isophotes; here, foreground stars in the field of view are clearly seen.

The absolute brightness were determined from comparison with the brightness from Hodge et al.
(1990) for several nebular regions. Sources 37b, c, e, and $g$ in the list of these authors were used for the flux calibration; i.e., the brightest and faintest regions were excluded.

Constructing the monochromatic image allowed us to clearly distinguish regions of weak $\mathrm{H}_{\alpha}$ emission from the background far outside the bright nebular core. As a result, we have been able for the first time to clearly show that these outer weak regions form a giant bipolar shell structure around the WO star 


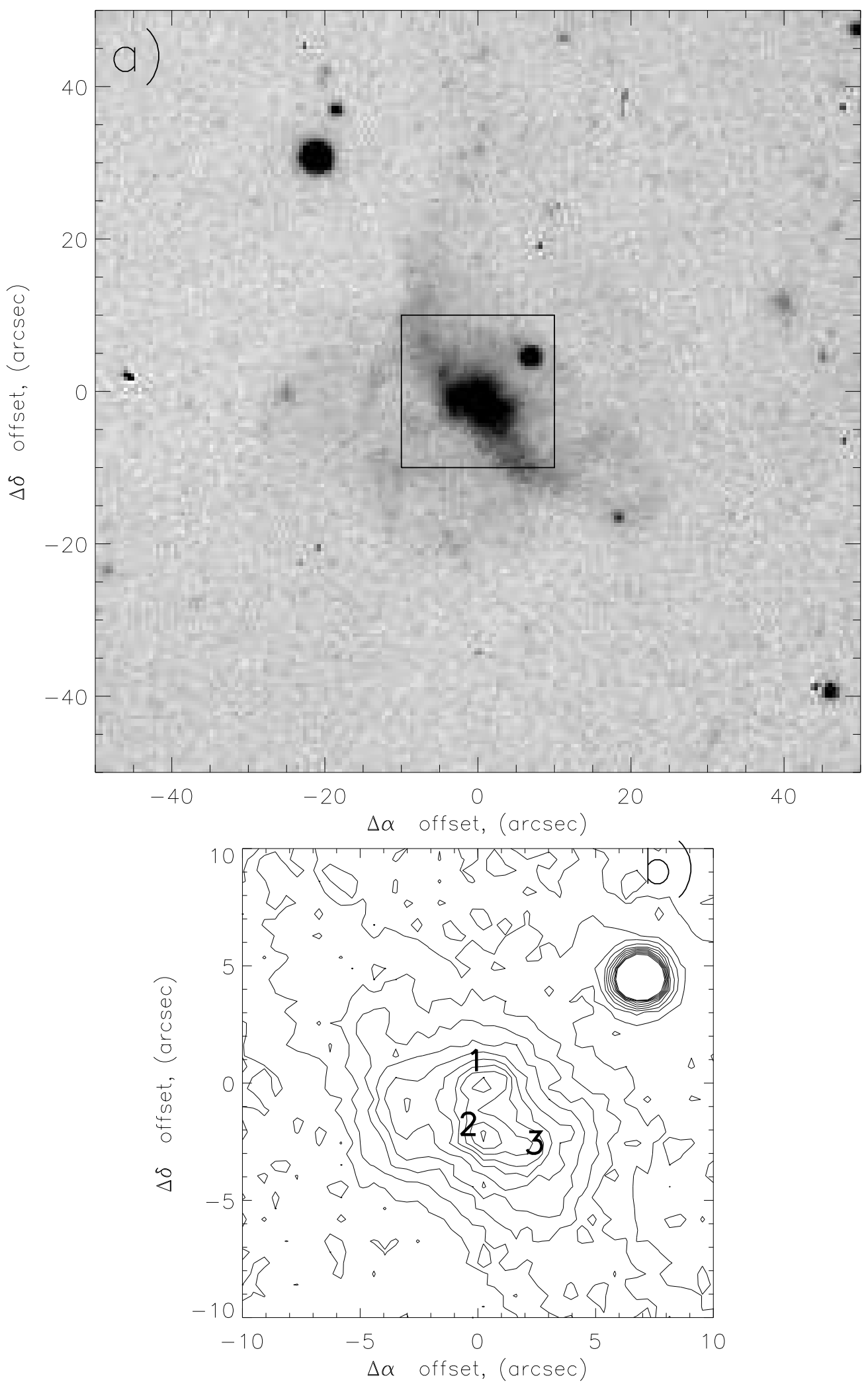

Figure 2: The narrow-band $\mathrm{H}_{\alpha}(\mathrm{a}, \mathrm{b})$ and [OIII] $(\mathrm{c}, \mathrm{d})$ images of the nebula obtained with the 4-m KPNO telescope. The numbers denote three compact sources; source N1 is most likely the WO star. The square encloses the region whose isophotes are shown below. 
and the bright S3 core. The two shells forming this bipolar structure are indicated by the dashed line in Fig. 1.

According to our measurements, the sizes of the three components (the bright core and the two outer shells) are the following: angular sizes of $26 \times 20^{\prime \prime}$ or linear sizes of $83 \times 66 \mathrm{pc}$ for the bright nebula S3; $35 \times 24^{\prime \prime}$ or $112 \times 77 \mathrm{pc}$ for the outer southeastern shell; and $(58-60)^{\prime \prime} \times(67-70)^{\prime \prime}$ or $(186-192) \times(214-224)$ pc for the outer northwestern shell.

We give the sizes along the major and minor axes of each structure for a distance of $660 \mathrm{kpc}$, as inferred by Saha et al. (1992)

\subsection{Narrow-Band $\mathbf{H}_{\alpha}$ and [OIII] Images}

Figure 2 shows the $\mathrm{H}_{\alpha}$ (Figs. 2a, 2b) and [OIII] (Figs $2 \mathrm{c}, 2 \mathrm{~d}$ ) images of the nebula obtained with the 4-m KPNO telescope. The southeastern outer shell and part of the northwestern shell at the very "base" near the bright nebular are seen in these figures. Both shells exhibit a distinct filamentary structure; in general, the filaments in the two lines are morphologically identical.

Three compact features unresolvable in the $6 \mathrm{~m}$ telescope monochromatic image because of the poor seeing on the observing night are clearly seen in the region of the bright S3 core. In all probability, the brightest of them (N1 in Fig. 2) is the WO star. The remaining features are most likely compact knots of gas, because, as follows from a comparison of Figs. 2b and $2 \mathrm{~d}$, their relative brightnesses in the two lines are different ( $\mathrm{N} 2$ is brighter than $\mathrm{N} 3$ in $\mathrm{H}_{\alpha}$ and weaker than the latter in $[\mathrm{OIII}])$. To elucidate the nature of these compact sources requires spectroscopic observations at a sufficiently good seeing.

\subsection{Kinematics}

Figure 3 shows the measured velocities at the line peak and the line FWHMs as obtained from our Fabry-Perot interferometric observations. In the data reduction, the line was fitted by a Gaussian; the line width was corrected for the instrumental profile width by assuming that the latter was also Gaussian. In order to find evidence for expansion of the outer bipolar shell, we constructed the distributions of line velocity and FWHM along two cuts designated, respectively, "X" and "Y" in Fig. 1. The band along the "X" axis is $60^{\prime \prime}$ wide, so the entire bipolar structure falls within it; the ( $15^{\prime \prime}$ wide) band along the "Y" axis covers predominantly the bright central S3 core and, in part, the weaker emission at the bases of the two outer shells.
A comparison of the line velocities and FWHMs along the two cuts leads us to the following conclusions:

1. The scatter of velocities of the line peak is systematically larger in both outer shells than that in the central bright $\mathrm{S} 3$ core. Indeed, the velocities in the central region $\left(-10^{\prime \prime} \div+10^{\prime \prime}\right)$ along the " $\mathrm{X}$ " axis in Fig. 1 vary in the range from -190 to $-255 \mathrm{~km} \mathrm{~s}^{-1}$; the velocities in the southeastern shell vary in the range from -165 to $-295 \mathrm{~km} \mathrm{~s}^{-1}$; and the range of velocities in the extended northwestern shell is largest: from -150 to $-300 \mathrm{~km} \mathrm{~s}^{-1}$.

As we see from the cut along the "Y" axis in Fig. 3b, the scatter of velocities in the bright S3 core is even smaller: velocities from -200 to $-230 \mathrm{~km} \mathrm{~s}^{-1}$ are observed in a band within $\pm 13^{\prime \prime}$ of the center (in accordance with the core size; see Fig. 1). At large distances from the center, the scatter increases because of the contribution of emission from the bases of both outer shells.

2. The line width in both shells also exceeds appreciably the line width in the central core. As we see from Fig. $3 \mathrm{c}$, the line width in the same central region $\left( \pm 10^{\prime \prime}\right)$ is no larger than $240 \mathrm{~km} \mathrm{~s}^{-1}$; in the southeastern shell, it reaches $330 \mathrm{~km} \mathrm{~s}^{-1}$; and the broadest line up to $390 \mathrm{~km} \mathrm{~s}^{-1}$ is observed in the northwestern shell.

The two observed patterns of behavior suggest the presence of systematic high-velocity motions in the outer bipolar shell structure. Based on these data, we may take an expansion velocity $\geq 50 \mathrm{~km} \mathrm{~s}^{-1}$ for the southeastern shell and $\geq>70 \mathrm{~km} \mathrm{~s}^{-1}$ for the northwestern shell as a rough estimate. The two values are a lower limit; the underestimate can be more significant for the northwestern shell, because here we observe mainly the emission from the periphery, where the effect of geometrical projection reduces the observed radial velocities.

Evidently, observations with a higher spectral and angular resolution are desirable to accurately estimate the expansion velocity. It should be borne in mind, however, that such an "erratic" velocity distribution - from slow bright knots to the most rapid weak features - is observed in most expanding supernova remnants and nebulae around the sources of strong stellar wind, which were studied with a high angular and spectral resolution (Lozinskaya,1986 and 1992). Indeed, the nebulae are irregular in shape and clumpy in structure; accordingly, the effect of geometrical projection changes erratically the observed radial velocity. During the passage of a shock wave, the gas of dense bright knots accelerates weakly, while 


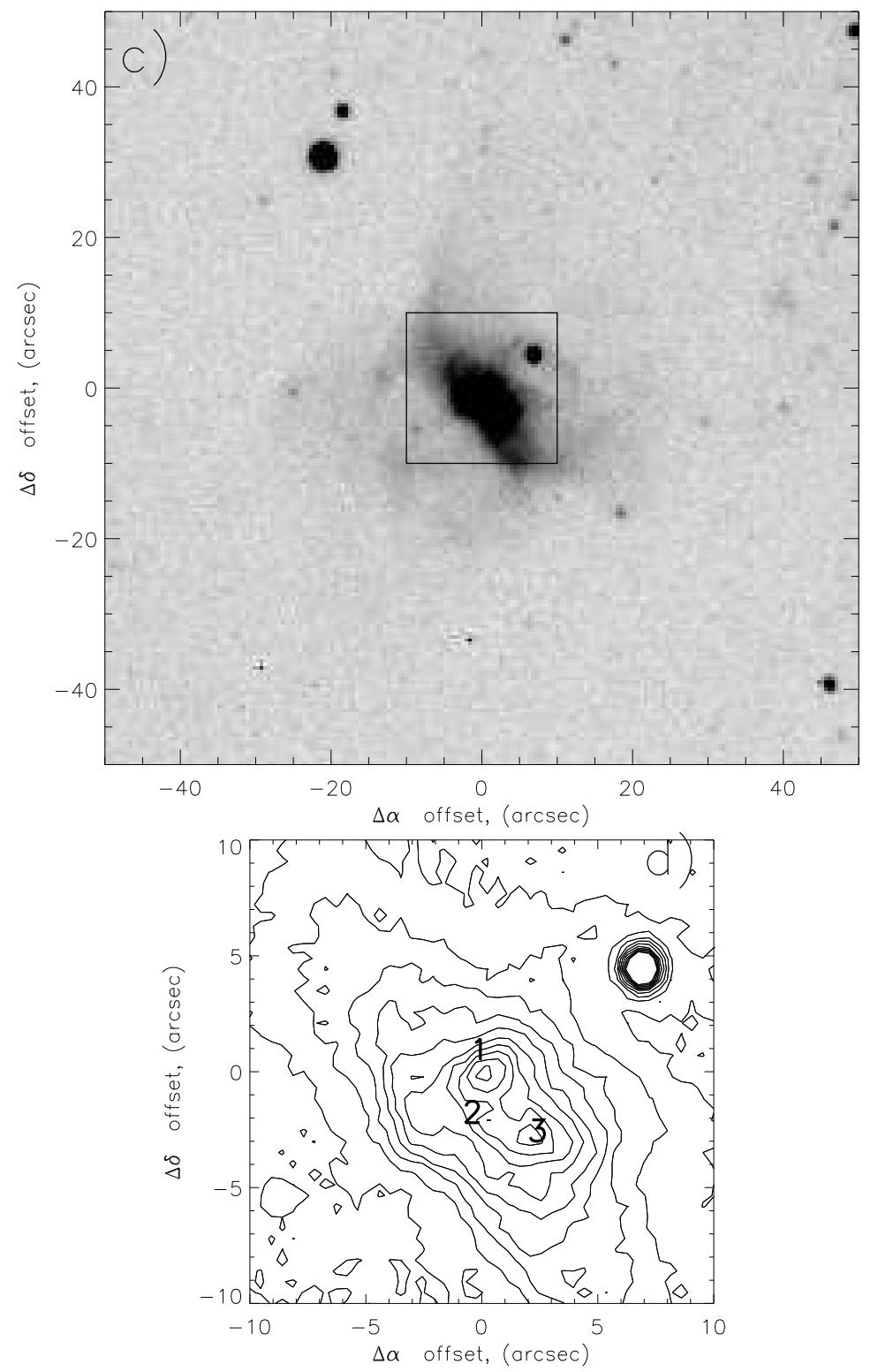

Figure 2. continued

the gas of lower density accelerates more strongly. For these reasons, the entire set of radial velocities, ranging from the gas at rest to the shock velocity, is commonly observed in real expanding shells. Higher resolution observations of the nebula around the WO star in IC 1613 could therefore reveal the same erratic distribution of line velocities and FWHMs.

According to our measurements, the mean veloc- 

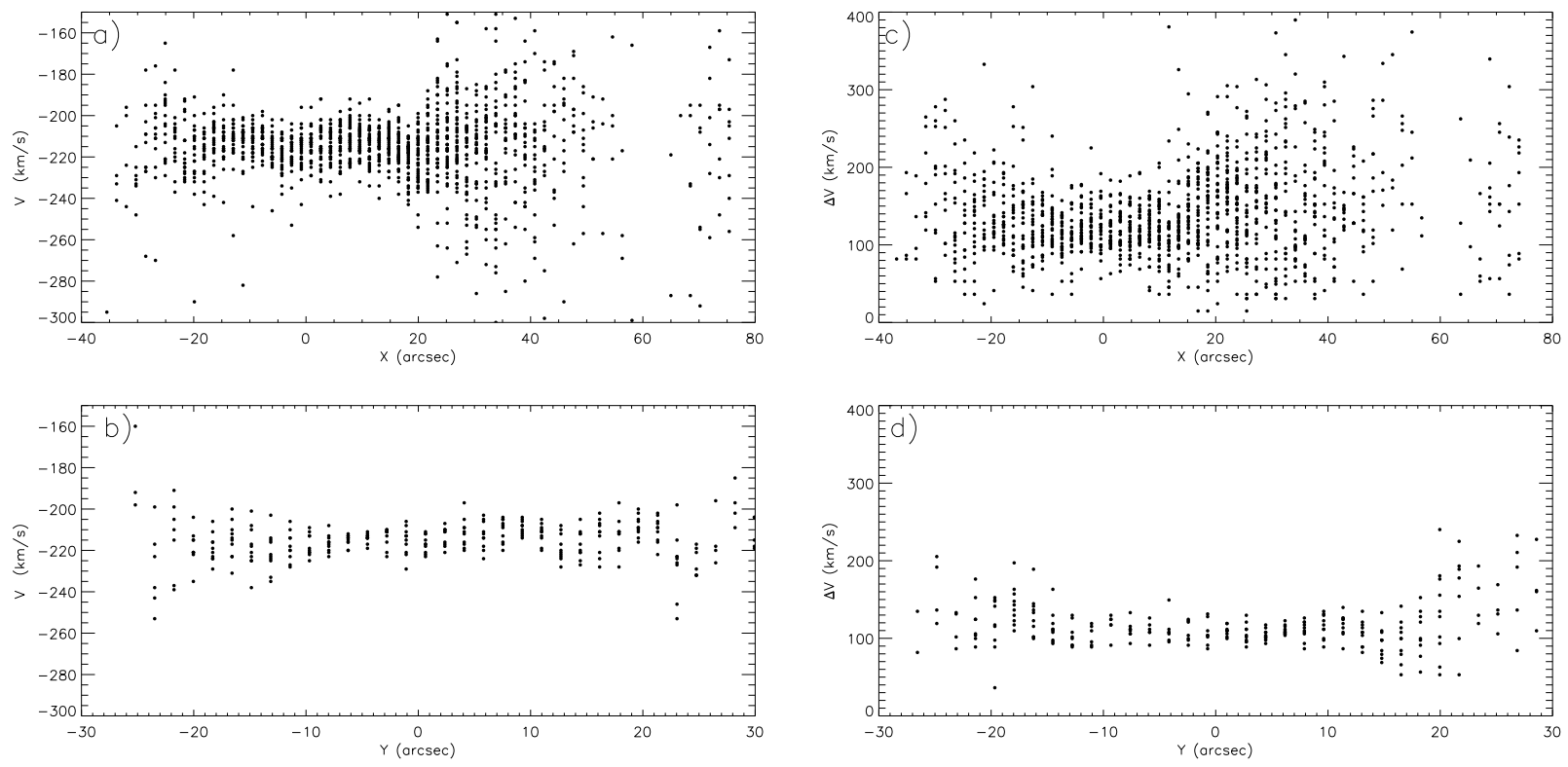

Figure 3: The measured velocity at line peak and the line FWHMs from our Fabry-Perot interferometric observations: (a) $\mathrm{V}(\mathrm{Hel})$ in a $60^{\prime \prime}$ wide band along the "X"-axis (see Fig. 1); (b) $\mathrm{V}(\mathrm{Hel}$ ) in a $15^{\prime \prime}$ wide band along the "Y"-axis; (c) and (d) the line FWHMs in the same bands along the "X" and "Y" axes, respectively.

ity of the bright part of the nebula, where the expansion effect is marginal, is $V(H e l)=-216 \mathrm{~km} \mathrm{~s}^{-1}$. Given a possible systematic tie-in error of the absolute velocity measurements within $15-20 \mathrm{~km} \mathrm{~s}^{-1}$ (see Sect. 2), this value is in agreement with the measurements by Tomita et al.(1993): $\mathrm{V}(\mathrm{Hel})=$ $-235 \div-230 \mathrm{~km} \mathrm{~s}^{-1}$, as inferred from one spectrogram of S3, and with the measurements by Lake \& Skillman (1989) in the 21-cm radio line: the mean velocity $V(\mathrm{Hel})=-232 \mathrm{~km} \mathrm{~s}^{-1}$ in the near galactic region.

\section{Discussion}

The existence of an outer bipolar structure outside the bright core of the nebula S3 in IC 1613 was first suspected by one of us (T.A.L.) back in 1988 on the basis of trial narrow-band [OIII] images obtained at her request by M.A. Dopita with the 2.6m MSSS ANU telescope, which have not been published. This suspicion was completely confirmed in this study.

Lozinskaya (1997) suggested the break out (burst) of a strong stellar wind in two directions out of the dense gas layer forming the "main body" of the nebula as a possible explanation for the bipolar structure of the outer shells. Not only the morphology of the nebula but also its orientation relative to the extended region of $\mathrm{H}$ I deficiency, which is clearly seen in the HI distribution from the 21-cm line emission obtained by Lake \& Skillman (1989), argue for this model. The center of this "supercavity" in the $\mathrm{H}$ I distribution has the following coordinates: $\mathrm{RA}=$ $1^{h} 2^{m} 20^{s}$ and DEC $=1^{\circ} 51^{\prime}$; its size is $2-3^{\prime}(400-600$ pc). The formation of such a supercavity may be associated with the preceding activity of stars in the region. If the $\mathrm{H} \mathrm{I}$ deficiency is assumed to be caused by the sweeping up of gas, then the orientation of the nebula's main body along the boundary of the hypothetical swept-up H I "supershell", the southeastern shell in the region of a denser medium, and the extended weak northwestern shell in the medium of lower density inside the supercavity can be explained naturally.

The bipolar shell structure associated with the WO star in IC 1613 and its location relative to the H I supercavity are schematically shown in Fig. 4.

The analogy with an extensively studied galactic object also argues for the break out of a strong stellar wind into the region of reduced gas density: the bright shell swept up by the wind in a dense cloud and the "blister" produced by the wind burst through the cloud into a tenuous medium are also observed in the 


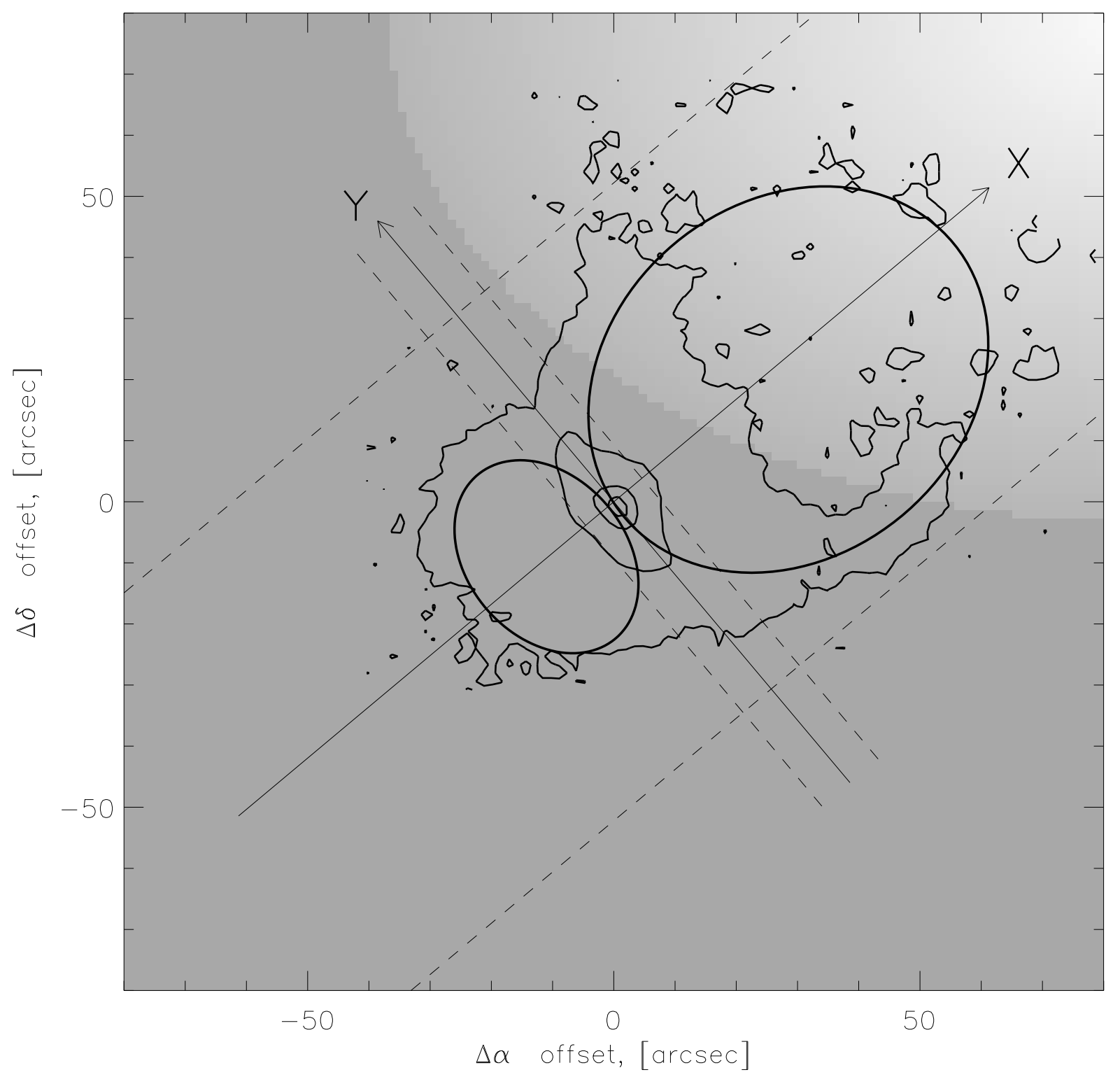

Figure 4: A scheme for the bipolar shell structure associated with the WO star and its location relative to the HI supercavity. The bipolar shell is represented by two ellipses. The dashed lines indicate the boundaries of the cuts along the "X" and "Y" axes (see Fig. 3). The $\mathrm{H}_{\alpha}$ isophotes as constructed from the $6 \mathrm{~m}$ telescope observations correspond to $0.04,0.5,1.5$ and $2.0 \times 10^{-15} \mathrm{erg} \mathrm{cm}^{-2} \mathrm{~s}^{-1} / \square^{\prime \prime}$ The shades of gray indicate the distribution of HI column density (the fit to the data from Lake \& Skillman, 1989). The transition from white to gray corresponds to column densities from 0.4 , to $2 \times 10^{2} 0 \mathrm{~cm}^{-2}$

nebula G2.4+1.4 associated with the star of the same type, WR 102 (Dopita \& Lozinskaya, 1990; Dopita et al.,1990).

An analysis of the parameters of the two shells associated with the WO star in IC 1613 yields the rough estimates that are in best agreement with the stan- dard model of wind effect on the interstellar medium. The size of both shells ( $R_{1} \simeq 56 \mathrm{pc}$ and $R_{2} \simeq 110 \mathrm{pc}$ ) is actually the only parameter that was reliably determined from observations. However, the fact that the ages of the two shells must be the same in the proposed model constraints significantly the range of 
possible values for the parameters.

The wind mechanical luminosity for the WO star in IC 1613 is $L_{w} \simeq 10^{38} \mathrm{erg} \mathrm{s}^{-1}$ (Kinsburgh \& Barlow, 1995). This estimate is rather crude, because only the wind velocity $\left(V_{w}=2850 \mathrm{~km} \mathrm{~s}^{-1}\right)$ was determined from observations, while the mass-loss rate $\left(2.9 \times 10^{-5} \mathrm{M}_{\odot} / \mathrm{yr}\right)$ was estimated indirectly from the relation between the HeII-line luminosity and the outflow rate for two WO stars.

The typical density of the interstellar medium in the vicinity of the star can be estimated with the same uncertainty.

First, based on the $\mathrm{H}_{\beta}$ emission from the brightest central part of S3 $6^{\prime \prime}$ in size, Davidson \& Kinman (1982) found the core density to be $N_{e}=8.5 \mathrm{~cm}^{-3}$. Second, using the total $\mathrm{H}_{\alpha}$ emission from the entire bright nebula, Kennicutt (1984) obtained a mean $N_{e} \simeq 1 \mathrm{~cm}^{-3}$. Third, Goss \& Lozinskaya (1995) estimated the mean density in the bright nebula S3 from the flux density of thermal radio emission to be $N_{e}=3.5 \mathrm{~cm}^{-3}$.

Given the dense knots that we detected in the central core, all the three estimates give a lower limit on the density in the bright nebula. These estimates refer to the densest region of the central nebula, where, judging by the radial-velocity field presented above, the gas was not accelerated by the stellar wind because of the high density.

The mean ambient gas density in a large region around the WO star can be obtained from the 21cm observations by Lake \& Skillman (1989). Their map of the H I column-density distribution gives $\mathrm{N}(\mathrm{HI}) \simeq(2-4) \times 10^{20} \mathrm{~cm}^{-2}$ for the region of interest. For a thickness of the galactic gas disk of $500-800 \mathrm{pc}$, this value corresponds to a mean density of $\simeq 0.1^{-3}$. We estimated the gas-disk thickness from the following considerations. According to the optical B surface-brightness profile given in the above paper, the stellar surface density at a distance $R \approx 6^{\prime}$ from the center is $\sigma_{*} \approx 2.5-3 M_{\odot} / \mathrm{pc}^{2}$, while the total surface density of stars and gas is $\sigma_{T} \approx 3.5-4 M_{\odot} / \mathrm{pc}^{2}$. The thickness of the gas disk can be roughly assumed to be $h_{0} \sim 1 / \sqrt{\sigma}_{T}$ (see, e.g. Zasov,1993). Since $\sigma_{T} \approx 50-70 M_{\odot} / \mathrm{pc}^{2}$ at a gasdisk thickness of $\approx 200 \mathrm{pc}$ in the solar neighborhood, we obtain $h_{0} \approx 500-800 \mathrm{pc}$ in the WO region for IC 1613.

All these estimates are rather crude, but they show that the initial density in the vicinity of the WO star is unlikely to fall outside the range from 0.01 to $5 \mathrm{~cm}^{-3}$.

The expected ages (in units of $10^{6}$ years) and expansion velocities for the two outer shells in the
Table 1: The expected ages and expansion velocities of the two outer shells in a medium of various densities

\begin{tabular}{|c|c|c|c|c|c|}
\hline$n_{o}, \mathrm{~cm}^{-3}$ & 5 & 1 & 0.1 & 0.01 & 0.001 \\
\hline$t_{6}\left(R_{1}\right)$ & 1.2 & 0.7 & 0.3 & 0.15 & 0.07 \\
$v_{1}, \mathrm{~km} \mathrm{~s}^{-1}$ & 28 & 46 & 100 & 218 & 460 \\
\hline$t_{6}\left(R_{2}\right)$ & 3.6 & 2.1 & 1.0 & 0.45 & 0.2 \\
$v_{2}, \mathrm{~km} \mathrm{~s}^{-1}$ & 18 & 30 & 63 & 140 & 300 \\
\hline
\end{tabular}

standard model of a wind-blown bubble (Castor et al.,1975; Weaver et al.,1977) are given in the table for various initial densities $n_{0}$ at $L_{w}=10^{38} \mathrm{erg} \mathrm{s}^{-1}$ and $R_{1} \simeq 56 \mathrm{pc}$ and $R_{2} \simeq 110 \mathrm{pc}$ derived above.

As we see from the table, similar age estimates for the two shells, depending on the radius, provided that the expected expansion velocity is close to the observed values $v_{1} \geq 50 \mathrm{~km} \mathrm{~s}^{-1}$ and $v_{2} \geq 70 \mathrm{~km} \mathrm{~s}^{-1}$, are obtained at an initial gas density $n_{o} \simeq 1-0.1 \mathrm{~cm}^{-3}$ for the brighter southeastern shell and $n_{o} \simeq 0.1-$ $0.01 \mathrm{~cm}^{-3}$ for the weaker extended northwestern shell. These ranges of ambient gas densities seem quite reasonable. The ratio of ambient gas densities in the two shells swept up by the wind is determined from the ratio of their sizes more accurately than the density itself: $n_{o, 1} / n_{o, 2}=\left(R_{2} / R_{1}\right)^{5} \simeq 20$.

The inferred age of the bipolar shell structure, $t_{1} \simeq(0.3-1) \times 10^{6}$ years, is close to the lifetime of a massive WR star and exceeds appreciably the duration of the final WO stage. Note that the age was estimated for the WO wind intensity. The wind intensity at the preceding WR stage was most likely lower, which increases still further the age estimate for the shell. Thus, we conclude that the unique bipolar structure was most likely produced by the wind from the central star at the stage preceding WO. The WO superwind, which switches on inside the previously formed structure, is an additional source of energy. Since the shock wave triggered by the strong WO wind propagates in a medium of very low density inside the existing bipolar shells, the "secondary" shells swept up by them rapidly reach the denser regions at the boundary. The additional compression of gas by the shock wave triggered by the superwind gives rise to a filamentary structure, which is clearly seen in Figs. 3a and 3b in the southeastern shell and at the base of the northwestern shell.

\section{Conclusion}

Our $\mathrm{H}_{\alpha}$ observations of the nebula associated with the WO star in the galaxy IC 1613 using a scanning 
Fabry-Perot interferometer on the $6 \mathrm{~m} \mathrm{SAO}$ telescope clearly revealed a giant bipolar shell structure outside the bright nebula S3. The sizes of the shell southeast of the bright nebula are $112 \times 77 \mathrm{pc}$; the northwestern shell is twice as large: $(186-192) \times(214-224)$ pc. The object's kinematics has been studied for the first time. We found evidence for expansion of both shells; the possible expansion velocities of the southeastern and northwestern shells are no less than 50 and $70 \mathrm{~km} \mathrm{~s}^{-1}$, respectively. Using the 4-m KPNO telescope (narrowband $\mathrm{H}_{\alpha}$ and [OIII] images), we detected a filamentary structure of both shells and several compact features in the S3 core.

We propose a self-consistent model for the formation of the giant bipolar structure by the wind from the central WO star located at the boundary of a dense supercavity in the galactic H I distribution.

Acknowledgements. This study was supported by the Russian Foundation for Basic Research (project no. 98-02-16032) and the Program "Astronomy" (project no. 1.3.1.2). We wish to thank M. Dopita, who provided unpublished [OIII] images of the region, J. Boulesteix for the opportunity to use interference filters, and the $6 \mathrm{~m}$ telescope committee for allocating observing time.

\section{References}

Armandroff, T. E. \& Massey, P., 1985, ApJ, 291, 685

Barlow. M.J., Hummer V., 1982, IAU Symp.99, "WolfRayet Stars: Observations, Physics, \& Evolution" ( de Loore C. W. H. \& Willis A. J. eds), 387

Boulesteix, J., 1993, "ADHOC Reference Manual" (Observatoire de Marseille, Marseille)

Castor, J., McCray, R., \& Weaver, R., 1975, ApJ, 200, L107

D’Odorico, S., \& Rosa, M., A \& A,1982, 105, 410

Davidson, K., \& Kinman, T. D., 1982, PASP, 94, 634

Dodonov, S. N., Vlasyuk, V. V., \& Drabek, S. V., 1995, "Fabry-Perot Interferometer. User's Guide", (SAO, Nizhnii Arkhyz)

Dopita, M.A., McGregor, P. J., Lozinskaya, T. A., \& Rawlings, S.J., 1990, ApJ,351, 563

Dopita, M.A., \& Lozinskaya, T.A., 1990, ApJ, 359, 419

Garnett, D. R., Kennicutt, R. C., Chu, Y.-H., \& Skillman, E. D., 1991, ApJ, 373, 458

Goss W. M., \& Lozinskaya, T. A., 1995, ApJ, 439, 637

Hodge, P.,Lee, M. G., \& Gurwell, M., 1990, PASP, 102, 1245

Hunter, D. A., Hawley, W. N., \& Gallagher, J. S., 1993, AJ, 106, 1797

Kennicutt, R. C., 1984, ApJ, 287, 116

Kinsburgh, R. L., \& Barlow, M. J., 1995, A \& A, 295, 171

Lake, G., \& Skillman, E.D., 1989, AJ, 98, 1274

Lozinskaya, T. A., 1986, "Supernovae and Stellar Wind:
Interaction with Galactic Gas", (Nauka, Moscow)

Lozinskaya, T. A., 1992, "Supernovae and Stellar Wind in the Interstellar Medium", (AIP, New York)

Lozinskaya, T.A.,1997, Astrophys. Space Sci., 252, 199

Maeder, A., \& Meynet, G., A \& A, 1989, 210, 155

Melnik J., \& Heydari-Malayeri, M., 1991, IAU Symp. 143, "WR Stars and Interrelations with Other Massive Stars in Galaxies, (Van der Hucht K. A. \& Hidayat B. ets), 409

Polcaro, V. F., Viotti R., Rossi, C., \& Norci, L.,1992, A \& A, 265,563

Polcaro, V. F., Giovanelli, F.,Manchanda, R. K., et al., A \& A, 1991, 252, 590

Saha, A., Freedman, W. L., Hoessel, J. G., \& Mossman, A. E., 1992, AJ, 104, 1072

Sandage, A. R., 1971, ApJ, 166, 13

Smith, H. E., 1975, ApJ,199, 591

Tomita, A., Ohta, K., \& Saito, M., 1993, PASJ, 45, 693

Torres, A. V., Conti, P. S., \& P. Massey,1986, ApJ, 300, 379

Weaver, R., McCray, R., Castor, J., et al., 1997, ApJ, 218, 377

Zasov, A. V., 1993, "Physics of Galaxies" (MSU, Moscow, 1993, in russuain). 